S.I.S.S.A. 101/93/EP

\title{
EFFECTIVE THEORY OF A CHIRAL SUPERFLUID
}

\author{
Pietro Donatis and Roberto Iengo \\ International School for Advanced Studies, I-34014 Trieste, Italy and \\ Istituto Nazionale di Fisica Nucleare, INFN, Sezione di Trieste, Trieste, Italy
}

\begin{abstract}
We consider an effective Lagrangian describing a fluid living on two-dimensional planes. The fluid self-interacts through a Chern-Simons vector potential, whose field strength is proportional to the density fluctuation. This effective Lagrangian can be related to the Anyon mean field, but can also be considered more generally to describe a universality class of superfluids and, when charged, of superconductors. We study the relevant physical properties, including the spectrum, the chirality features appearing in the polarization of scattered EM waves, and the peculiar response under a magnetic field, i.e. a peculiar kind of anisotropic Meissner effect.
\end{abstract}




\section{Introduction and summary}

We study here the physical implications of an effective Lagrangian à la LandauGinzburg, describing a possible peculiar superfluid. We are interested in particular in the case when the superfluid is charged, behaving thus as a peculiar superconductor. The distinctive features of this superfluid are, first the fact that it lives on a two dimensional surface, and this is in common with various theoretical studies of layered superconductors (systems essentially made of two dimensional layers over which the supercurrent can flow). Also, many high $T_{c}$ superconductors appear to behave in this way.

The other, more peculiar, feature is chirality; that is the fact that the dynamics of this superfluid makes a distinction between some left and right handed behaviour. This is built in the defining effective Lagrangian, which breaks parity, although the consequences may not be immediate to see. In fact, the effective Lagrangian contains a peculiar selfinteraction expressed in terms of a Chern-Simons gauge field. One can thus phrase our investigation as a study of a universality class of some superfluids, which can be related to Anyon systems. Of course, one can also forget about Anyons, and consider the present superfluid per se as a possible physical system.

The main point about the Chern-Simons gauge field is that we assume that its field strength (also called "curvature") is determined by the request that it is proportional to the density fluctuation, i.e. the actual density at a given point minus the average density. This assumption makes the effective Lagrangian consistent, and, in particular, compatible with translational invariance.

Formally, the same effective Lagrangian has been introduced in refs. [1] [2] [3] [4], to provide a possible description of the Fractional Quantum Hall Effect (FQHE). Here, on the contrary, we do not assume a strong, uniform external magnetic field, orthogonal to the surface, like in the FQHE. Thus, our investigation is rather devoted to the superfluidity and the possible superconductivity properties. Essentially the same effective Lagrangian also been previously considered in ref. [5] in a generic context of Anyon superconductivity. Our treatment of the problem is however basically different from what discussed in ref. $[5]$.

We devote an appendix (Appendix A) to the relation of our effective Lagrangian with

the translationally invariant version of the Anyon Mean Field theory developed in refs. [6] [7] [8] [9].

In Section 2 we begin by discussing the effective Lagrangian and the spectrum of the excitations it describes, first by a perturbative treatment and then by studying also the vortex excitations. We find a spectrum with a finite gap, and this gap is of course the same 
as that found in ref. [4]. However there is an important difference in the range of values of a parameter, having the meaning of a selfinteraction coupling constant. Here we are interested in a range of values for which we show that the "roton" (or vortex) part of the spectrum is above the gap, contrary to the case relevant for the FQHE considered in ref. [4]. In fact, our range of values of the parameter is such that when the effective Lagrangian is compared with Anyon Mean Field (Appendix A), all the excitations correspond to the inter Landau levels ones, rather than the intra Landau levels as relevant for FQHE. Thus, in our case, the spectrum resembles the one of an ordinary superfluid, shifted up by the gap amount, as shown in Fig. 1.

We then discuss in Section 3 the chirality properties. In particular we show that a superfluid layer has an optical activity, in that it transmits only circularly polarized electromagnetic waves of a definite handedness, in a range of frequencies near to the above mentioned gap. Qualitatively, those frequencies are in the microwave/infrared regime (for reasonable values of the parameters), where it would be measurably difficult to detect, and we show that the effect disappears at high frequencies.

In Section 4 we discuss the magnetic properties of the chiral superfluid, discussing the possibility of the Meissner effect in the bulk of a sample made of a stack of many layers (see Fig. $2 a$ and $2 b$ ). We find a very different behaviour, depending on the orientation of the magnetic field relative to the layers. If the field is orthogonal to the layers, case $a$, then the system behaves as a type II superconductor with a finite penetration length. We show it by considering both the penetration from an edge and also studying magnetic vortices. In order to get the correct result one has to include in the computation the electrostatic forces, which of course are present and important in a charged superfluid in general, but are particularly essential in our case.

In the case $b$, where the magnetic field is parallel to the layers, the behaviour is very different. One has to make a further distinction between to cases: the field, which is parallel to the layers' plane, can either try to penetrate $i$ ) in the direction parallel to the layers or else $i i$ ) in the direction orthogonal to the layers. In the first case $i$ ), the behaviour is determined, like in the layered superconductors studied in ref. [10] by the amount of the interlayer Josephson coupling. Namely if the Josephson coupling is small the penetration length in the interlayer space can be large. This is the same as for ordinary superconductors.

In the second case $i$ ) the chiral superconductor behaves differently from an ordinary one. We show, by an order of magnitude argument, that in this case the penetration length, in the direction orthogonal to the layers, is not finite and independent of the size of the sample. Rather, it grows with the power $1 / 3$ of the sample's size, apart from logarithmic corrections. 
Summarizing, the effective Lagrangian we have studied describes a possible physical system which is rich of rather interesting structures.

\section{Effective theory}

We take as a starting point the following non relativistic effective Lagrangian density in two space and one time dimensions:

$$
\mathcal{L}=i \phi^{*} \partial_{0} \phi-\frac{1}{2 m}|\vec{D} \phi|^{2}-g|\phi|^{4}=i \phi^{*} \partial_{0} \phi-\mathcal{H}
$$

here $\phi(\vec{x}, t)$ is a non relativistic complex field which plays the rôle of order parameter, related to the density by

$$
\rho=|\phi|^{2}
$$

We assume a fixed total number of particles $N$, therefore we keep fixed $N=\int d^{2} x|\phi|^{2}$.

$\vec{D}$ is the covariant derivative $\vec{D}=\vec{\nabla}-i \overrightarrow{\tilde{A}}$ and $\tilde{A}$ is the "fluctuation" of a CS gauge potential determined by:

$$
\vec{\nabla} \wedge \overrightarrow{\tilde{A}}=\frac{2 \pi}{k} \delta \rho \quad \delta \rho=\rho-v^{2}=|\phi|^{2}-v^{2} \quad v^{2}=<\rho>
$$

As a consequence of the conservation of the number of particles we have consistently:

$$
\int d^{2} x \vec{\nabla} \wedge \overrightarrow{\vec{A}}=0
$$

One can compare equation (2.3) with the standard definition of the CS field describing Anyons, where the CS field strength is proportional to the density. Our effective Lagrangian (2.1) is meant to describe the physics of the fluctuations over the constant density, and therefore the dynamical effects of the CS fluctuations as in equation (2.3).

In Appendix A we show how to relate the Lagrangian (2.1) to the Anyon Mean Field theory.

For convenience we choose to change the zero of the energy spectrum adding to the Hamiltonian density the constant quantity $-g v^{4}$. So that in $(2.1) \mathcal{H}$ becomes:

$$
\mathcal{H}=\frac{1}{2 m}|(\vec{\nabla}-i \overrightarrow{\widetilde{A}}) \phi|^{2}+g\left(|\phi|^{4}-v^{4}\right)=\frac{1}{2 m}|(\vec{\nabla}-i \overrightarrow{\widetilde{A}}) \phi|^{2}+g(\delta \rho)^{2}
$$

here we used the fact that $\int d^{2} x \delta \rho=0$ in the Hamiltonian. 


\subsection{Small deformations approach}

The first analysis we want to perform on Lagrangian (2.1) is based on a small deformation approach. Let us take the following parameterization:

$$
\phi=f e^{i \theta}
$$

Since the integral over all the plane of $\delta \rho(\vec{x}, t)$ is zero, we can write it as the divergence of some quantity, and we choose:

$$
\delta \rho(x)=2 v \vec{\nabla} \cdot \vec{u}(\vec{x}, t)
$$

furthermore we have the gauge freedom to take $\vec{u}(\vec{x}, t)$ irrotational, that is

$$
\vec{\nabla} \wedge \vec{u}=\partial_{x} u_{y}-\partial_{y} u_{x}=0
$$

Then we have from (2.3) and (2.7):

$$
\vec{\nabla} \wedge \overrightarrow{\tilde{A}}=\frac{4 \pi v}{k} \vec{\nabla} \cdot \vec{u}
$$

this equation can be solved by:

$$
\tilde{A}_{x}=-\frac{4 \pi v}{k} u_{y} \quad \tilde{A}_{y}=\frac{4 \pi v}{k} u_{x}
$$

Notice that $\vec{\nabla} \cdot \overrightarrow{\tilde{A}}=0$.

With the new parameterization the Lagrangian becomes (neglecting higher orders):

$$
\mathcal{L}=2 v \theta \partial_{0}(\vec{\nabla} \cdot \vec{u})-\frac{1}{2 m} v^{2}(\vec{\nabla} \theta)^{2}-\frac{1}{2 m}\left(\frac{4 \pi v^{2}}{k}\right)^{2} \vec{u}^{2}-\frac{1}{2 m}(\triangle \vec{u})^{2}-4 g v^{2}(\vec{\nabla} \cdot \vec{u})^{2}
$$

Performing the variation with respect to $\theta$ we get:

$$
2 v \partial_{0}(\vec{\nabla} \cdot \vec{u})+\frac{v^{2}}{m} \vec{\nabla} \cdot \vec{\nabla} \theta=0 \quad \Rightarrow \quad \frac{v^{2}}{m} \vec{\nabla} \theta+2 v \dot{\vec{u}}=0
$$

which can be regarded as the continuity equation:

$$
\partial_{0} \rho+\vec{\nabla} \cdot(\rho \vec{V})=0
$$

provided we identify the velocity $\vec{V}=\frac{\vec{\nabla} \theta}{m}$. 
Inserting back this equation in the Lagrangian (2.11) we get:

$$
\mathcal{L}=2 m \dot{\vec{u}}^{2}-\frac{1}{2 m}\left(\frac{4 \pi v^{2}}{k}\right)^{2} \vec{u}^{2}-\frac{1}{2 m}(\triangle \vec{u})^{2}-8 g v^{2}(\vec{\nabla} \cdot \vec{u})^{2}
$$

so we have managed to write the Lagrangian as a kinetic part minus a potential part. Taking $\vec{u}=\vec{u}_{0} e^{i(E t+\vec{p} \cdot \vec{x})}$ we find the spectrum of the energy:

$$
2 m E^{2}=\frac{1}{2 m}\left(\frac{4 \pi v^{2}}{k}\right)^{2}+\frac{1}{2 m}\left(p_{x}^{2}+p_{y}^{2}\right)^{2}+8 g v^{2}\left(p_{x}^{2}+p_{y}^{2}\right)
$$

Here we are neglecting a contribution to the energy coming from an electrostatic interaction between fluctuations, which will play an essential rôle in the next chapter. The piece to be added to the Hamiltonian density is

$$
\frac{e^{2}}{8 \pi} \delta \rho(\vec{x}) \int d^{2} x^{\prime} \frac{1}{\left|\vec{x}-\vec{x}^{\prime}\right|} \delta \rho\left(\vec{x}^{\prime}\right)
$$

its contribution to the r.h.s. of equation $(2.15)$ is $+e^{2} v^{2}|\vec{p}|$.

The minimum of the energy, for $p=0$, is not zero, so we have a gap:

$$
E(0)=\frac{2 \pi}{m k} v^{2} \equiv \mathcal{E}
$$

Also currents can be computed:

$$
\begin{aligned}
& J_{x}=\frac{1}{2 m i}\left[\phi^{\dagger} D_{x} \phi-\phi\left(D_{x} \phi\right)^{\dagger}\right]=\frac{v^{2}}{m}\left(\partial_{x} \theta+\frac{4 \pi}{k} v u_{y}\right) \\
& J_{y}=\frac{1}{2 m i}\left[\phi^{\dagger} D_{y} \phi-\phi\left(D_{y} \phi\right)^{\dagger}\right]=\frac{v^{2}}{m}\left(\partial_{y} \theta-\frac{4 \pi}{k} v u_{x}\right)
\end{aligned}
$$

which, in a compact form, can be rewritten as:

$$
J_{i}=-2 v\left(\dot{u}_{i}-\mathcal{E} \epsilon_{i j} u_{j}\right)
$$

This current exhibits the chiral property of our system. The chiral effects appear for $p \rightarrow 0$. To see this let us parameterize $\vec{u}(\vec{x}, t)$ as follows:

$$
u_{x}(\vec{x}, t)=u_{0 x} \cos \left(E t+\varphi_{x}\right) \quad u_{y}(\vec{x}, t)=u_{0 y} \cos \left(E t+\varphi_{y}\right)
$$

then if $E=\mathcal{E}$ i.e. $p=0$, the currents (2.17) can be rewritten:

$$
J_{x}=J_{0} \cos \left(\mathcal{E} t+\varphi_{0}\right) \quad J_{y}=J_{0} \sin \left(\mathcal{E} t+\varphi_{0}\right)
$$


where $J_{0}$ and $\varphi_{0}$ are constants depending on $\vec{u}_{0}$.

Notice that this is a circularly polarized current with a definite direction of rotation. It can be easily seen that, since such direction depends on the sign of $k$, it reverses performing a parity transformation. In our case $k$ is fixed since the Lagrangian (2.1) describe a physical system which is not parity invariant.

\subsection{Vortex excitations}

Now we go beyond the small deformations and study the vortex solutions, i.e. vortex configurations, that minimize the energy:

$$
E=\int d^{2} r\left\{\frac{1}{2 m}|\vec{D} \phi|^{2}+g\left(v^{2}-|\phi|^{2}\right)^{2}\right\}
$$

We will follow the method of reference [11] [12] [13] based on the classical work of Bogomol'nyi [14]. Vortices are classical static solutions of the form:

$$
\phi(r, \theta)=f(r) e^{i n \theta} \quad \lim _{r \rightarrow \infty} f(r)=v
$$

here $n$ is a topological invariant corresponding to the vorticity, that is how many times the vortex winds round; the elementary vortex has $|n|=1$.

Now since in polar coordinates we have (we call here simply $A$ what we called $\tilde{A}$ in $(2.1)$ ):

$$
B=\frac{1}{r} \partial_{r}\left(r A_{\theta}\right)-\partial_{\theta} A_{r}
$$

we can choose for the gauge field the form, see equation (2.3):

$$
\left\{\begin{array}{l}
A_{r}=0 \\
A_{\theta}=-\frac{2 \pi}{k} \frac{1}{r} \int_{0}^{r} d r^{\prime} r^{\prime}\left(v^{2}-f^{2}\left(r^{\prime}\right)\right)
\end{array}\right.
$$

With this choice the total energy becomes:

$$
E=\int d^{2} r\left\{\frac{1}{2 m}\left[\left(\partial_{r} f\right)^{2}+\frac{1}{r^{2}}\left(n-r A_{\theta}\right)^{2} f^{2}\right]+g\left(v^{2}-|\phi|^{2}\right)^{2}\right\}
$$

from this equation we get the following requirement for the energy to be finite:

$$
\lim _{r \rightarrow \infty} A_{\theta}(r)=\frac{n}{r} \rightarrow 0
$$


For our particular case this yields:

$$
\frac{2 \pi}{k} \int_{0}^{\infty} d r r\left[v^{2}-f^{2}(r)\right]=-n
$$

which is nothing but the quantization of the magnetic flux in integer factors of $2 \pi$.

The problem now is to minimize the total energy; for this purpose it is useful the following identity [15]:

$$
|\vec{D} \phi|^{2}=\left|\left(D_{x} \pm i D_{y}\right) \phi\right|^{2} \pm m \vec{\nabla} \wedge \vec{J} \pm B|\phi|^{2}
$$

here $\vec{J}$ is the usual current

$$
\vec{J}=\frac{1}{2 m i}\left[\phi^{\dagger} \vec{D} \phi-\phi(\vec{D} \phi)^{\dagger}\right]
$$

Then we have:

$$
E=\int d^{2} r\left\{\frac{1}{2 m}\left|\left(D_{x} \pm i D_{y}\right) \phi\right|^{2} \pm \frac{1}{2} \vec{\nabla} \wedge \vec{J} \pm \frac{1}{2 m} B|\phi|^{2}+g\left(v^{2}-|\phi|^{2}\right)^{2}\right\}
$$

Due to (2.3), we can rewrite the third term as:

$$
\frac{1}{2 m} B|\phi|^{2}=\frac{\pi}{m k}\left(v^{2}-|\phi|^{2}\right)^{2}+\frac{v^{2}}{2 m} \vec{\nabla} \wedge \vec{A}
$$

so we are left with:

$$
E=\int d^{2} r\left\{\frac{1}{2 m}\left|\left(D_{x} \pm i D_{y}\right) \phi\right|^{2} \pm \frac{1}{2} \vec{\nabla} \wedge\left(\vec{J} \pm \frac{v^{2}}{m} \vec{A}\right)+\left(g \pm \frac{\pi}{m k}\right)\left(v^{2}-|\phi|^{2}\right)^{2}\right\}
$$

Let us compute the contribution of the second term:

$$
\frac{1}{2} \int d^{2} r \vec{\nabla} \wedge\left(\vec{J}+\frac{v^{2}}{m} \vec{A}\right)=\frac{1}{2} r \int d \theta \frac{v^{2}}{m} \frac{n}{r}=\frac{\pi v^{2}}{m} n
$$

Therefore the total energy is:

$$
E= \pm \frac{\pi v^{2}}{m} n+\int d^{2} r\left\{\frac{1}{2 m}\left|\left(D_{x} \pm i D_{y}\right) \phi\right|^{2}+\left(g \pm \frac{\pi}{m k}\right)\left(v^{2}-|\phi|^{2}\right)^{2}\right\}
$$

From this expression we can learn several things. Firstly we note that the energy $E$ is positive definite, see equation (2.21). We will always study the case where $g \geq \frac{\pi}{m k}$.

If one wants to relate (2.1) to the Anyon mean field we notice that the value of $g$ which reproduces the Anyon mean field energy is $g=\frac{\pi}{m}\left(1-\frac{1}{k}\right)$ (see Appendix A) and we see that $\frac{\pi}{m}\left(1-\frac{1}{k}\right) \geq \frac{\pi}{m k}$ with $k \geq 2$. 
Therefore the integral on the r.h.s. of equation (2.34) is positive or zero. So we derive the inequality:

$$
E \geq \frac{\pi v^{2}}{m}|n| \geq \frac{2 \pi}{m k} v^{2}=\mathcal{E}
$$

So we have found that also the "big" deformations have energies over the gap $\mathcal{E}$.

Let us now study the special case $g=\frac{\pi}{m k}$ and $n<0$, where the bound (2.35) is saturated. In this case the equations simplify because the energy becomes:

$$
E=\frac{\pi v^{2}}{m}|n|+\frac{1}{2 m} \int d^{2} r\left\{\left|\left(D_{x}-i D_{y}\right) \phi\right|^{2}\right\}
$$

which is minimal for:

$$
\left(D_{x}-i D_{y}\right) \phi=0 .
$$

Following Jackiw and Weinberg [13] we call equation (2.37) self-dual condition.

Let us now solve the equation (2.37). In polar coordinates it is written:

$$
\frac{\partial f}{\partial r}+\frac{n}{r} f-A_{\theta} f=0 .
$$

If we introduce the auxiliary variable

$$
a=-n+r A_{\theta}
$$

with the properties:

$$
\begin{gathered}
a(0)=-n>0 \\
\lim _{r \rightarrow \infty} a(r)=0
\end{gathered}
$$

equation (2.38) is equivalent to the system:

$$
\left\{\begin{array}{l}
\partial_{r} f=\frac{1}{r} a f \\
\partial_{r} a=-\frac{2 \pi}{k} r\left(v^{2}-f^{2}\right)
\end{array}\right.
$$

This non-linear couple of differential equations has no analytic solution; so we will study its asymptotic behaviours and then solve it numerically.

As $r \rightarrow \infty$ the equations can be linearized defining $F=(v-f) \rightarrow 0$ and we get an asymptotic solution in terms of Bessel functions:

$$
\left\{\begin{array}{l}
F(r)=\alpha K_{0}\left(\sqrt{\frac{4 \pi}{k}} v r\right) \\
a(r)=\alpha \sqrt{\frac{4 \pi}{k}} r K_{1}\left(\sqrt{\frac{4 \pi}{k}} v r\right)
\end{array}\right.
$$


here

$$
\lim _{z \rightarrow \infty} K_{0}(z)=\sqrt{\frac{\pi}{2 z}} e^{-z} \quad K_{1}(z)=-K_{0}^{\prime}(z)
$$

and $\alpha$ is some constant.

For $r \rightarrow 0$ we have:

$$
\left\{\begin{array}{l}
f(r)=A_{n} r^{n}+\cdots \\
a(r)=n-\frac{\pi}{k} v^{2} r^{2}+\cdots
\end{array}\right.
$$

$A_{n}$ being some constant.

We solved the equation numerically (with DO2GAF-NAG Fortran Library Routine) and verified the agreement of the solution with the previous discussion.

In the case of $n>0$, the equations cannot be reduced to first order and the bound cannot

be saturated. We will study this case by a variational method which, in agreement with (2.35), confirms that the vortex energy for $n=+|n|$ is in fact higher than that for $n=-|n|$. This fact should be expected since the opposite signs of $n$ are connected by a parity transformation, but our Lagrangian density (2.1) is not parity invariant, so vortices that differ for the sign of $n$ cannot have the same energy.

\subsubsection{Currents}

In this subsection we compute the electric current density and the total current due to the vortex for $n<0$

From (2.29) using equation (2.38) we get:

$$
\left\{\begin{array}{l}
J_{r}=0 \\
J_{\theta}=-\frac{e}{m} f \partial_{r} f
\end{array}\right.
$$

The total current $I$ passing in the plane is equal to

$$
\begin{gathered}
I=\int d \vec{s} \cdot \vec{J}=\int_{0}^{\infty} d r J_{\theta}(r)=-\frac{e}{m} \int_{0}^{\infty} d r f \partial_{r} f=-\frac{e}{m} \int_{0}^{\infty} d r \frac{d}{d r} f^{2}= \\
=-\frac{e}{m}\left[f^{2}(\infty)-f^{2}(0)\right]=-\frac{e}{m} v^{2}
\end{gathered}
$$

This current can be interpreted as the Hall current of a QHE!

To see this fact let us consider the following Hamiltonian:

$$
H=|n| \frac{\pi v^{2}}{m}+\frac{1}{2 m} \int d^{2} r\left\{|\vec{D} \phi|^{2}+e B|\phi|^{2}\right\}
$$


where $B=-\frac{2 \pi}{k e}\left(v^{2}-|\phi|^{2}\right)$. It is straightforward to prove that this Hamiltonian is equivalent to our original Hamiltonian with $g=\frac{\pi}{m k}$. In this form we can interpret the second term in curly brackets in $(2.47)$ as an interaction term between the electric potential $V=\frac{1}{2 m} B$ and the electric charge density $e|\phi|^{2}$. To such a potential will correspond the electric field $\vec{E}=-\vec{\nabla} V$ which in polar coordinates reads:

$$
\left\{\begin{array}{l}
E_{r}=-\frac{\partial V}{\partial r}=-\frac{1}{2 m} \frac{\partial B}{\partial r}=-\frac{2 \pi}{m k e} f \partial_{r} f=-\frac{2 \pi}{k e^{2}} J_{\theta} \\
E_{\theta}=-\frac{1}{r} \frac{\partial V}{\partial \theta}=0
\end{array}\right.
$$

From the first of the $(2.48)$ we read the value of the Hall conductivity $\sigma=\frac{J_{\theta}}{E_{r}}$ :

$$
\sigma=k \frac{e^{2}}{2 \pi}
$$

\subsubsection{Variational method}

We have found exact vortex-like solutions obeying the self-dual condition (2.37) for $n<0$. In order to further investigate the general case we try to find the vortices in a variational way. This can be done with the following ansatz for $\tilde{A}$ :

$$
r \tilde{A}_{\theta}(r)=n\left[1-\left(1+\frac{p^{2} r^{2}}{2}\right) e^{-\omega r^{2}}\right]
$$

and minimizing the energy (2.25) with respect to the parameters $p$ and $\omega$. Correspondently we get for $f(r)$, see equation (2.22), imposing the condition $f(0)=0$ which yields the relation $p^{2}=2 \omega+\frac{n k v^{2}}{2 \pi}$ between the parameters,

$$
f^{2}(r)=v^{2}-\left(v^{2}-\frac{n k}{2 \pi} p^{2} \omega r^{2}\right) e^{-\omega r^{2}}
$$

In the minimization program we have taken $|n|=1, g=\frac{\pi}{m}\left(1-\frac{1}{k}\right), k=2$ to compare the result with the minimum find above, see equation (2.35).

For $n<0$ we have found $E_{\text {min }}=6.44 \frac{v^{2}}{2 m}$ which is very close $(6.44 \simeq 2 \pi)$ to the exact result, i.e. the gap $\mathcal{E}$.

For $n>0$ we have found $E_{\text {min }}=20.64 \frac{v^{2}}{2 m}$ which is quite above the gap.

Notice that the variation in the number of the particles due to these vortices is:

$$
\delta N=\int d^{2} r \delta \rho=\int d^{2} r\left(f^{2}(r)-v^{2}\right)=\int d^{2} r\left[\left(\frac{n k}{2 \pi} p^{2} \omega r^{2}-v^{2}\right) e^{-\omega r^{2}}\right]=n k
$$

So the total number of particles is preserved only if the total vorticity is zero. 


\subsection{Summary of the spectrum}

We can conclude saying that all the excitations of the system lie over the gap.

Recalling equation (2.15), with the correction coming from the electrostatic interaction between excitations (see the comment after equation (2.15)), we see that for small $p$ we have:

$$
E(p) \simeq \mathcal{E}+\frac{e^{2} k}{8 \pi}|\vec{p}|
$$

For larger values of $p$ it is possible to have a roton excitation [1] [2] [3] [4] which can be explained as a Coulomb interaction between vortices.

In fact, the Coulomb interaction gives an additional positive contribution to the energy, namely the electrostatic energy due to the density fluctuation of a charged fluid. This electrostatic energy is obviously positive for any density fluctuation and in particular for the vortex-antivortex configurations. Therefore, in conclusion, the roton part of the spectrum will correspond to an energy higher than the lower bound of a vortex-antivortex configuration, that is twice the gap $\mathcal{E}$. In Fig. 1 we report the qualitative plot of the energy spectrum versus the momentum. (In the case considered in reference [4] the vortices are assumed to appear in the lowest Landau level of a Hall system, whereas the gap of the small deformations is due to excitations to the higher Landau levels. Thus, in this case the vortices can have an energy less that the gap. The case studied in reference [4] would correspond in our formalism to $g<\frac{\pi}{m k}$. In our case instead we take $g \geq \frac{\pi}{m k}$, see the discussion of section 2.2).

Fig. 1 


\section{Chiral property}

In this section we study how the chirality properties of our superfluid show up in the optical activity. Similar studies have appeared in the literature for other kinds of $T$ and $P$-breaking theories, see in particular ref. [16]. Let us consider an electromagnetic wave incoming on a thin layer of superfluid, perpendicularly to the plane of the layer. We begin the analysis studying how the electromagnetic potential couples with our system on the plane. The small deformation Lagrangian, equation (2.14), is modified adding the incoming electromagnetic potential $\vec{A}$ :

$$
\mathcal{L}=2 v \theta \vec{\nabla} \cdot \dot{\vec{u}}-\frac{1}{2 m} v^{2}\left(\vec{\nabla} \theta-\frac{4 \pi v^{2}}{k} \tilde{\vec{u}}+e \vec{A}\right)^{2}-\frac{1}{2 m}(\triangle \vec{u})^{2}-4 g v^{2}(\vec{\nabla} \cdot \vec{u})^{2}
$$

here $\tilde{\vec{u}}$ is the dual of $\vec{u}$ in the sense that $\tilde{u}_{i}=\epsilon_{i j} u_{j} . \vec{A}$ is a two dimensional vector lying in the plane. All the derivative operators act here on the two dimensional coordinates on the plane.

Performing the variation with respect to $\theta$ we find:

$$
\frac{\delta \mathcal{L}}{\delta \theta}=2 v \vec{\nabla} \cdot \dot{\vec{u}}+\frac{v^{2}}{m} \vec{\nabla} \cdot\left(\vec{\nabla} \theta-\frac{4 \pi v^{2}}{k} \tilde{\vec{u}}+e \vec{A}\right)=0
$$

From (2.8) we have $\vec{\nabla} \cdot \tilde{\vec{u}}=0$. Decomposing $\vec{A}=\vec{A}_{L}+\vec{A}_{\perp}$, where by definition $\vec{\nabla} \cdot \vec{A}_{\perp}=0$, we have:

$$
\vec{\nabla} \theta=-\frac{2 m}{v} \dot{\vec{u}}-e \vec{A}_{L}
$$

Then the lagrangian (3.1) can be rewritten as:

$$
\begin{gathered}
\mathcal{L}=-2 v \dot{\vec{u}} \cdot \vec{\nabla} \theta-\frac{1}{2 m} v^{2}\left(\vec{\nabla} \theta+e \vec{A}_{L}\right)^{2}-\frac{1}{2 m} v^{2}\left(e \vec{A}_{\perp}-\frac{4 \pi v^{2}}{k} \tilde{\vec{u}}\right)^{2}-\frac{1}{2 m}(\triangle \vec{u})^{2}- \\
-4 g v^{2}(\vec{\nabla} \cdot \vec{u})^{2}
\end{gathered}
$$

Now we introduce the following parameterization $\vec{A}=\vec{\nabla} \varphi+\tilde{\vec{\nabla}} \psi$, that is $A_{i}=\partial_{i} \varphi+\epsilon_{i j} \partial_{j} \psi$. Thus equation (3.4)(3.3)becomes:

$$
\begin{gathered}
\mathcal{L}=2 m \dot{\vec{u}}^{2}+2 v e \dot{\vec{u}} \cdot \vec{\nabla} \varphi-2 m \mathcal{E}^{2} \vec{u}^{2}+2 v \mathcal{E} \vec{u} \cdot \vec{\nabla} \psi-\frac{e^{2} v^{2}}{2 m}(\vec{\nabla} \psi)^{2}-\frac{1}{2 m}(\triangle \vec{u})^{2}- \\
-4 g v^{2}(\vec{\nabla} \cdot \vec{u})^{2}
\end{gathered}
$$

Taking $\vec{u}=\vec{u}_{0} e^{i(\omega t+\vec{p} \cdot \vec{x})}$ we find:

$$
\mathcal{L}=2 m\left[\omega^{2}-\mathcal{E}^{2}-g(p)\right] \vec{u}^{2}+2 v \vec{u} \cdot(i \omega \vec{\nabla} \varphi+\mathcal{E} \vec{\nabla} \psi)-\frac{e^{2} v^{2}}{2 m}(\vec{\nabla} \psi)^{2}
$$

here $g(p)$ stands for the $p$-dependent terms in (2.15).

By performing the functional integration over $\vec{u}$ and remembering that $\vec{\nabla} \varphi=\frac{\vec{p} \cdot \vec{A}}{p^{2}} \vec{p}$ and 
$\vec{\nabla} \psi=\frac{\epsilon_{i j} A_{i} p_{j}}{p^{2}} \vec{p}$, we get a quadratic piece in $A$

$$
\mathcal{L}_{e f f}=-\frac{1}{2} A^{\dagger} \hat{\mathcal{L}} A
$$

where:

$$
\hat{\mathcal{L}}=\frac{e^{2} v^{2}}{m} \frac{1}{\omega^{2}-\mathcal{E}^{2}-g(p)} \frac{1}{p^{2}} \times\left(M_{1}+M_{2}\right)
$$

The matrices $M_{1}$ and $M_{2}$ are given by:

$$
\begin{gathered}
M_{1}=\left(\begin{array}{cc}
\left|i p_{x} \omega+p_{y} \mathcal{E}\right|^{2} & \left(i p_{x} \omega+p_{y} \mathcal{E}\right)^{*}\left(i p_{y} \omega-p_{x} \mathcal{E}\right) \\
\left(i p_{y} \omega-p_{x} \mathcal{E}\right)^{*}\left(i p_{x} \omega+p_{y} \mathcal{E}\right) & \left|i p_{y} \omega-p_{x} \mathcal{E}\right|^{2}
\end{array}\right) \\
M_{2}=\frac{e^{2} v^{2}}{m p^{2}}\left(\begin{array}{cc}
p_{y}^{2} & -p_{x} p_{y} \\
-p_{x} p_{y} & p_{x}^{2}
\end{array}\right)
\end{gathered}
$$

Here we have introduced the following notation:

$$
A=\left(\begin{array}{c}
A_{x} \\
A_{y}
\end{array}\right)
$$

$\mathcal{L}_{\text {eff }}$ is the contribution to the total lagrangian coming from the interaction between the incoming wave and the two dimensional chiral fluid. Now we suppose $\vec{A}$ propagating in the $z$ direction and the plane situated at $z=0$; if we write $A=A(z) e^{i(\omega t+\vec{p} \cdot \vec{x})}$, with $A(z)$ discussed below, the total lagrangian is:

$$
\mathcal{L}(A)=\frac{1}{2} \omega^{2} a^{2}-\frac{1}{2}\left[p^{2} A^{2}+\left(\partial_{z} A\right)^{2}\right]-\frac{1}{2} A^{\dagger} \hat{\mathcal{L}} A \delta(z)
$$

from equation (3.12) we extract the equation of motion for $A$ :

$$
\partial_{z}^{2} A+\left(\omega^{2}-p^{2}\right) A-\hat{\mathcal{L}} A \delta(z)=0
$$

For $A(z)$ we have:

$$
A(z)= \begin{cases}\alpha_{-} e^{i k z}+\beta_{-} e^{-i k z} & \text { if } z<0 \\ \alpha_{+} e^{i k z} & \text { if } z>0\end{cases}
$$

Imposing continuity at $z=0$ for $A(z)$ and its derivative, we get:

$$
\alpha_{+}=\frac{2 i k}{2 i k-\hat{\mathcal{L}}} \alpha_{-}, \beta_{-}=\alpha_{+}-\alpha_{-}=\frac{\hat{\mathcal{L}}}{2 i k-\hat{\mathcal{L}}} \alpha_{-}
$$

From equation (3.15) we can read the matrices of reflection and transmission:

$$
R=\frac{\frac{\hat{\mathcal{L}}}{2 i k}}{1-\frac{\hat{\mathcal{L}}}{2 i k}} \quad T=\frac{1}{1-\frac{\hat{\mathcal{L}}}{2 i k}}
$$


Notice that $R=T-1$ and $|T|^{2}+|R|^{2}=1$ as it should.

In particular we are interested in the coefficient of transmission in the limit when $\omega \rightarrow \mathcal{E}$. In this case only $M_{1}$ in equation (3.12) is important. If we compute the inverse of the matrix $1-\frac{1}{2 i k} \hat{\mathcal{L}}$ we get, taking for simplicity $\vec{p}=(0, p)$ :

$$
T=\frac{1}{1-2 i(q+s)-2 q s}\left(\begin{array}{cc}
1-i q & -q \\
q & 1-i(q+2 s)
\end{array}\right)+O(1 / q)
$$

here we have set $q=-\frac{1}{2 k} \frac{e^{2} v^{2}}{m} \frac{\mathcal{E}^{2}}{\omega^{2}-\mathcal{E}^{2}}$ and $s=-\frac{1}{2} \frac{1}{2 k} \frac{e^{2} v^{2}}{m}$.

The limit $\omega \rightarrow \mathcal{E}$ corresponds to $q \rightarrow \infty$ that is:

$$
T=\frac{1}{2} \frac{i}{i+s}\left(\begin{array}{cc}
1 & -i \\
i & 1
\end{array}\right)
$$

Now let us consider the following parameterization:

$$
\begin{aligned}
& A_{x}=R e\left(\cos \theta e^{-i \omega t}\right)=\cos \theta \cos \omega t \\
& A_{y}=\operatorname{Re}\left(\sin \theta e^{i \varphi} e^{-i \omega t}\right)=\sin \theta \cos (\varphi-\omega t)
\end{aligned}
$$

we can see that the circular polarization is for $\theta=\frac{\pi}{4}$ and $\varphi=\frac{\pi}{2}$, so that:

$$
A_{x}=\frac{\sqrt{2}}{2} \cos \omega t \quad A_{y}=\frac{\sqrt{2}}{2} \sin \omega t
$$

in our complex formalism this corresponds to:

$$
A=\left(\begin{array}{c}
\cos \theta \\
\sin \theta e^{i \varphi}
\end{array}\right) e^{-i \omega t}=\frac{\sqrt{2}}{2}\left(\begin{array}{l}
1 \\
i
\end{array}\right) e^{-i \omega t}
$$

Thus we see that $T$ projects a state in a circularly polarized one, that is we can write:

$$
T=\frac{1}{2} \frac{i}{i+s}|c\rangle\langle c|
$$

where

$$
|c\rangle=\left(\begin{array}{l}
1 \\
i
\end{array}\right)
$$

That is, at the resonance $\omega \rightarrow \mathcal{E}$ our chiral planar system behaves like a perfect polarizer. Finally note that if $\omega \rightarrow \infty$ we get:

$$
\hat{\mathcal{L}} \rightarrow \frac{e^{2} v^{2}}{m}\left(\begin{array}{ll}
1 & 0 \\
0 & 1
\end{array}\right)
$$

so we have lost any polarizing effect. 
If conversely we take $\omega \rightarrow 0$ and $p \rightarrow 0$ we get $\hat{\mathcal{L}}=0$, so there is no more coupling between the electromagnetic wave and the planar system.

\section{Coupling with an external magnetic field}

\subsection{Magnetic field orthogonal to the layers}

\subsubsection{Meissner effect}

In this section we test our chiral charged superfluid with an external magnetic field, and study its Meissner effect.

Up to now we have been considering a two dimensional system; now we are going to study an effect which is essentially three-dimensional. Therefore we suppose to have a multilayered bulk of many two-dimensional thin films separated by a spacing $d$ (for a review on the properties of the layered superconductors see, for example [17]). We further suppose that at the edge of the bulk there is a uniform, constant magnetic field orthogonal to the layers' plane as in Fig. 2a.

Fig. 2 
In this three dimensional system we must take into account also an electrostatic contribution, (see the comment after equation (2.15)) and which will play an essential rôle here. This contribution is essentially due to an electric field $\vec{E}$ which comes from the fluctuations of the charged matter and obeys the Maxwell equation:

$$
\vec{\nabla} \cdot \vec{E}=e \delta \rho^{(3)}
$$

here $\delta \rho^{(3)}=\frac{\delta \rho}{d}$, is the three dimensional density.

In other words if there is a fluctuation $\delta \rho$ of matter the system will not be in electrostatic equilibrium anymore, for there will be some zones where there is lack of charged matter and others where there is abundance: $\vec{E}$ is the electric field resulting from this non-equilibrium situation.

So let us consider the following three-dimensional Hamiltonian density (in this section we have redefined $\overrightarrow{\tilde{A}} \rightarrow \vec{A}^{C S}$ to distinguish from $\vec{A}^{e m}$ ):

$$
\mathcal{H}=\frac{1}{2 m d}|\vec{D} \phi|^{2}+\frac{1}{2}\left(\vec{B}^{2}+\vec{E}^{2}\right)+\frac{g}{d}\left(\rho-v^{2}\right)^{2}
$$

here

$$
\vec{B}=\vec{\nabla} \wedge \vec{A}^{e m} \quad \vec{E}=-\vec{\nabla} A_{0}^{e m} \quad \vec{D}=\vec{\nabla}-i \vec{A}^{C S}-i e \vec{A}^{e m}
$$

Notice that the following equations hold:

$$
\left\{\begin{array} { l } 
{ \vec { \nabla } \cdot \vec { E } = \frac { e } { d } \delta \rho } \\
{ \vec { \nabla } \wedge \vec { E } = 0 }
\end{array} \quad \left\{\begin{array}{l}
\vec{\nabla} \cdot \vec{A}^{C S}=0 \\
\vec{\nabla} \wedge \vec{A}^{C S}=\frac{2 \pi}{k} \delta \rho
\end{array}\right.\right.
$$

here $\vec{E}$ is a three-dimensional vector whereas $\vec{A}^{C S}$ is two-dimensional. If we suppose that the matter distribution is constant in the $z$ direction, which means that we have exactly the same matter distribution in every layer, then we have no electric field orthogonal to the $x-y$ layers' plane. With this assumption equations (4.4) tell that $\vec{E}$ and $\vec{A}^{C S}$ are dual two-dimensional vectors, i.e. :

$$
E_{i}=\frac{k e}{2 \pi d} \epsilon_{i j} A_{j}^{C S}
$$

So we can write the hamiltonian as follows:

$$
\frac{H}{L_{z}}=\int d x d y\left\{\frac{1}{2 m d}|\vec{D} \phi|^{2}+\frac{1}{2}\left[\left(\vec{\nabla} \wedge \vec{A}^{e m}\right)^{2}+\frac{k^{2} e^{2}}{4 \pi^{2} d^{2}}\left(\vec{A}^{C S}\right)^{2}\right]+\frac{g}{d}\left(\rho-v^{2}\right)^{2}\right\}
$$

here we have performed the integration in $z$.

At this point we make one further assumption: we suppose that the external magnetic field is constant in $y$, which is the direction parallel to the edge of the bulk, so we can take $\vec{A}^{e m}(x)$ in the $y$ direction:

$$
\vec{A}^{e m}=\left(0, A^{e m}(x), 0\right)
$$


furthermore we still have the freedom to take also $\vec{A}^{C S}(x)$ in the $y$ direction:

$$
\vec{A}^{C S}=\left(0, A^{C S}(x), 0\right)
$$

In the gauge:

$$
\vec{\nabla} \cdot \vec{A}^{e m}=\vec{\nabla} \cdot \vec{A}^{C S}=0
$$

$A^{\text {em }}$ and $A^{C S}$ depend only on $x$. With these assumptions all quantities in (4.6) depend only on $x$, so we can perform the integration in $y$ and get:

$$
\begin{gathered}
\frac{H}{L_{y} L_{z}}=\int_{0}^{\infty} d x\left\{\frac{1}{2 m d}\left[\left|\partial_{x} \phi\right|^{2}+\left|e A^{e m}+A^{C S}\right|^{2} \rho\right]+\frac{1}{2}\left(\partial_{x} A^{e m}\right)^{2}+\right. \\
\left.+\frac{k^{2} e^{2}}{8 \pi^{2} d^{2}}\left(A^{C S}\right)^{2}+\frac{g}{d}\left(\rho-v^{2}\right)^{2}\right\}
\end{gathered}
$$

$L_{y}$ being the length of the edge of the bulk.

Now, before going into computational details, we want to spend some time analyzing qualitatively the reason why we expect a Meissner effect. To this end we will make some simplificatory assumptions. The first is to suppose that some external device is keeping constant the magnetic flux $\Phi_{0}=L_{y} l_{x} B$, where $L_{y}$ is the length of the edge of the sample and $l_{x}$ is the penetration length of the magnetic field, so that

$$
B l_{x}=\frac{\Phi_{0}}{L_{y}}=\varphi_{0}
$$

is given to the system from outside.

The second assumption we make is to suppose $B$ constant for $0<x<l_{x}$ and zero for $x>l_{x}$ whereas actually it is exponentially decreasing.

The third assumption we make is to take a constant value for the density $\rho=v^{2}$.

So we have for $0<x<l_{x}$ :

$$
B=\frac{\varphi_{0}}{l_{x}} \quad A^{e m}(x)=\frac{x}{l_{x}} \varphi_{0}
$$

For $x>l_{x}, A^{e m}$ is constant and it is possible to cancel it in the covariant $y$-derivative with the phase of $\phi$. Thus, the integration runs from 0 to $l_{x}$.

To test the meaningfulness of what we are doing let us see what happens for the well known case of the standard superconductor, i.e. with $\vec{A}^{C S}=0$; the hamiltonian (4.10) becomes:

$$
\frac{H}{L_{y} L_{z}}=\int_{0}^{l_{x}} d x\left\{\frac{1}{2} \vec{B}^{2}+\frac{1}{2 m d} e^{2}\left(A^{e m}\right)^{2} v^{2}\right\}=\frac{1}{2} \frac{\varphi_{0}^{2}}{l_{x}}+\frac{1}{6 m d} e^{2} v^{2} \varphi_{0}^{2} l_{x}
$$


Minimizing $H$ with respect to $l_{x}$ we find $l_{x}=\sqrt{\frac{3 m d}{e^{2} v^{2}}}$ which is indeed of the same order as the standard value of the penetration length for type II superconductors:

$$
\lambda=\sqrt{\frac{m}{e^{2} \rho^{(3)}}}
$$

Notice that in what we have done a fundamental rôle is played by the term quadratic in $A^{\text {em }}$, the "mass term" for the electromagnetic field.

In our case, with $\vec{A}^{C S} \neq 0$, this effect could be ruined by the possible cancellation $\vec{A}^{C S}=$ $-e \vec{A}^{e m}$ but, notice, we have also the electrostatic interaction term quadratic in $\vec{A}^{C S}$ which now plays the dominant rôle. We have:

$$
\frac{H}{L_{y} L_{z}}=\int_{0}^{l_{x}} d x\left\{\frac{1}{2} \vec{B}^{2}+\frac{1}{2} \vec{E}^{2}+\frac{1}{2 m d}\left(e A^{e m}+A^{C S}\right)^{2} v^{2}\right\}=\frac{1}{2} \frac{\varphi_{0}^{2}}{l_{x}}+\frac{k^{2} e^{4}}{24 \pi^{2} d^{2}} \varphi_{0}^{2} l_{x}
$$

Minimizing with respect to $l_{x}$ we get $l_{x}=\frac{2 \pi d \sqrt{3}}{k e^{2}}$ we see that we get a finite penetration length, so we expect to have Meissner effect.

Now we turn back to hamiltonian (4.10) and analyze it more quantitatively in the framework of the small deformation approach introduced in the previous chapter.

So we have, recalling the basic definitions, see equations (2.6), (2.7) and (2.10):

$$
\begin{gathered}
\phi=f \quad \partial_{x} \phi(x)=\partial_{x}^{2} u(x) \\
\delta \rho=2 v \partial_{x} u(x) \\
A^{C S}=\frac{4 \pi v}{k} u \quad g=\frac{\pi}{m}\left(1-\frac{1}{k}\right)
\end{gathered}
$$

Notice that in this one-dimensional case $\phi$ is real, so there is no phase.

The hamiltonian becomes:

$$
\begin{gathered}
\frac{H}{L_{y} L_{z}}=\frac{1}{d} \int_{0}^{\infty} d x\left\{\frac{1}{2 m}\left[\left(\partial_{x}^{2} u\right)^{2}+\left(e A^{e m}+\frac{4 \pi v}{k} u\right)^{2} v^{2}\right]+\right. \\
\left.+\frac{d}{2}\left(\partial_{x} A^{e m}\right)^{2}+\frac{2 v^{2} e^{2}}{d} u^{2}+\frac{4 \pi v^{2}}{m}\left(1-\frac{1}{k}\right)\left(\partial_{x} u\right)^{2}\right\}
\end{gathered}
$$

We now make the following ansatz:

$$
u(x)=u_{0} e^{-\lambda x} \quad A^{e m}(x)=A_{0} e^{-\lambda x}
$$


here $u_{0}$ and $A_{0}$ are the values at the edge of the bulk. The resulting equations for $u(x)$ and $A^{e m}(x)$ can be written in the form:

$$
\left(\begin{array}{cc}
\frac{\lambda^{4}}{2 m}-\frac{4 \pi v^{2}}{m}\left(1-\frac{1}{k}\right) \lambda^{2}+\frac{8 \pi^{2} v^{4}}{m k^{2}}+\frac{2 e^{2} v^{2}}{d} & \frac{2 \pi v^{3} e}{m k} \\
\frac{2 \pi v^{3} e}{m k} & \frac{e^{2} v^{2}}{2 m}-\frac{d}{2} \lambda^{2}
\end{array}\right)\left(\begin{array}{c}
u \\
A^{e m}
\end{array}\right)=0
$$

Putting to zero the determinant of this matrix we get:

$$
\begin{gathered}
\frac{d}{4 m} \lambda^{6}-\left[\frac{2 \pi v^{2} d}{m}\left(1-\frac{1}{k}\right)+\frac{e^{2} v^{2}}{4 m^{2}}\right] \\
\lambda^{4}+\left[\frac{8 \pi v^{4} d}{2 m k^{2}}+e^{2} v^{2}+\frac{2 \pi e^{2} v^{4}}{m^{2}}\left(1-\frac{1}{k}\right)\right] \lambda^{2}- \\
-\frac{e^{4} v^{4}}{m d}=0
\end{gathered}
$$

If we take the following typical values for the parameters (as an order of magnitude we take $m$ to be the electron mass):

$$
d=1 \AA \quad e^{2}=\frac{4 \pi}{137} \quad v^{2}=4 \cdot 10^{-3} \AA^{-2} \quad m=250 \AA^{-1} \quad k=2
$$

we get the solutions:

$$
\lambda_{1}=1.21 \cdot 10^{-3} \quad \lambda_{2,3}=0.56 \pm i 0.54 \equiv \alpha \pm i \beta
$$

We can approximately compute analytically the smallest eigenvalue $\lambda_{1}$ by rewriting equation (4.22) in an approximate form taking only the leading terms:

$$
\lambda^{2}\left\{\frac{d}{4 m} \lambda^{4}-\left[\frac{2 \pi v^{2} d}{m}\left(1-\frac{1}{k}\right)\right] \lambda^{2}+e^{2} v^{2}\right\}=\frac{e^{4} v^{4}}{m d}
$$

for small $\lambda^{2}$ we get the solution:

$$
\lambda^{2}=\frac{e^{4} v^{4}}{m d} \frac{1}{e^{2} v^{2}}=\frac{e^{2} v^{2}}{m d}
$$

which, compared with equation (4.14), is exactly the expression for the inverse of the square of the standard penetration length, and, corresponds numerically to $\lambda_{1}$ in equation (4.24).

Let us note that by solving (4.22) in the limit of very small $e^{2}$ we would find:

$$
\lambda=\frac{k e^{2}}{\sqrt{4 \pi} d}\left(1+O\left(e^{2}\right)\right)
$$

which, apart for inessential numerical factors, is equal to the inverse of the $l_{x}$ found minimizing (4.15). Indeed the qualitative analysis leading to (4.15) was meant in the limit of 
a very small electrostatic interaction. Actually this is not the case. For instance, with the values given in (4.23) we get $\frac{8 \pi v^{4} d}{2 m k^{2}} \ll e^{2} v^{2}$, and (4.26) follows.

The general solution for $u(x)$ is the linear combination:

$$
u(x)=u_{1} e^{-\lambda_{1} x}+u_{2} e^{-\lambda_{2} x}+u_{3} e^{-\lambda_{3} x}
$$

Imposing the reality condition $u^{*}(x)=u(x)$ we can write:

$$
u(x)=u_{1} e^{-\lambda_{1} x}-e^{-\alpha x}\left(u_{1} \cos \beta x-w \sin \beta x\right)
$$

giving for $\delta \rho$ :

$$
\delta \rho=2 v \partial u=2 v\left[-\lambda_{1} u_{1} e^{-\lambda_{1} x}+e^{-\alpha x}\left(\left(\alpha u_{1}+\beta w\right) \cos \beta x+\left(\beta u_{1}-\alpha w\right) \sin \beta x\right)\right]
$$

Requiring $\rho$ to vanish at the edge, i.e. $\delta \rho(0)=-v^{2}$ we can determine the value of $w$ :

$$
w=-\frac{v}{2 \beta}-\frac{u_{1}}{\beta}\left(\alpha-\lambda_{1}\right)
$$

The following relation holds between $A^{e m}(x)$ and $u(x)$ :

$$
A^{e m}(x)=\frac{4 \pi v^{3} e}{m k d} \frac{1}{\lambda^{2}-\frac{e^{2} v^{2}}{m d}} u(x)
$$

We then compute $B=\partial_{x} A^{e m}$.

Imposing $B(0)=B_{0}$ we get a value for $u_{1}$, the only parameter still undetermined. If we substitute the values of the parameters we see that the exponential behaviour of $B$ is controlled by the first eigenvalue, i.e. the penetration length is $\frac{1}{\lambda_{1}}=826.45 \AA$. We can compare this value to the numerical value of the standard penetration length (4.14) which is $825.57 \AA$.

It is seen numerically that the behaviour of $\delta \rho$ is controlled by the other eigenvalues, i.e. the coherence length is $\frac{1}{\alpha}=1.78 \AA$.

Notice that the penetration length is about 400 times the coherence length, so our system behaves like a type II superconductor.

\subsubsection{Vortices}

In this section we study, with a variational method, the vortices in presence of an external electromagnetic field orthogonal to the layers' plane.

These vortices have an origin different from the vortices studied in section 1.2. Those were originated by fluctuations of the CS magnetic field, i.e. of the matter density from the 
mean value. The present ones are instead the standard well known vortex configurations of superconductors of type II between the two critical temperatures. They consist of small regions of the specimen of normal behaviour, all surrounded by a superconducting region, where the external magnetic field penetrates completely and uniformly. There are superconducting currents flowing around the vortices. Furthermore there is a penetration of the magnetic field from the vortex region to the surrounding superconducting region (Meissner effect).

As we saw in the previous section the hamiltonian is:

$$
\frac{H}{L_{z}}=\int d^{2} r\left\{\frac{1}{2 m d}|\vec{D} \phi|^{2}+\frac{1}{2} \vec{B}^{2}+\frac{k^{2} e^{2}}{8 \pi^{2} d^{2}}\left(A^{C S}\right)^{2}+\frac{g}{d}\left(\rho-v^{2}\right)^{2}\right\}
$$

where we have included the electrostatic effect, that is the third term on the r.h.s., as in equation (4.6).

We make the following ansatz:

$$
\begin{aligned}
& \phi=f(r) e^{i n \theta} \\
& e r A_{\theta}^{e m}=n\left(1-e^{-\mu r^{2}}\right) \quad A_{r}^{e m}=0 \\
& r A_{\theta}^{C S}=\frac{2 \pi}{k} \int_{0}^{r} d r^{\prime} r^{\prime}\left[f^{2}\left(r^{\prime}\right)-v^{2}\right]=\frac{\pi}{k} v^{2} r^{2} e^{-\omega r^{2}} \quad A_{r}^{C S}=0
\end{aligned}
$$

from the last one we easily get:

$$
f^{2}(r)=v^{2}-v^{2}\left(1-\omega r^{2}\right) e^{-\omega r^{2}}
$$

Notice that our ansatz is such that we have quantization of the flux of the external magnetic field, differently from the vortices studied previously when it was the flux of the CS magnetic field to be quantized. Notice also that since the vorticity is no longer connected with the CS field now, differently from the vortices studied before, it is possible to have an isolated vortex or antivortex since $\int_{0}^{\infty} d r r\left[v^{2}-f^{2}(r)\right]=0$ as can be checked from the last of equations (4.34).

If one substitutes in (4.33) and minimizes, numerically, with respect to $\lambda$ and $\omega$, with the usual values of the parameters (4.23) one gets:

$$
\omega=0.101 \AA^{-2} \quad \mu=7.336 \cdot 10^{-7} \AA^{-2}
$$

for the case of the vortex $(n=1)$ and

$$
\omega=0.117 \AA^{-2} \quad \mu=7.337 \cdot 10^{-7} \AA^{-2}
$$


for the case of the antivortex $(n=-1)$.

From these results we get the values of the dimensions of the vortex and of the fluctuations of the magnetic field:

$$
\frac{1}{\sqrt{\omega}}=3.147 \AA \quad \frac{1}{\sqrt{\mu}}=1167.54 \AA
$$

for the vortex, and

$$
\frac{1}{\sqrt{\omega}}=2.924 \AA \quad \frac{1}{\sqrt{\mu}}=1167.46 \AA
$$

for the antivortex. Notice that for the vortex and for the antivortex we have different results, although very close to each other, as it should have been expected since our system is chiral.

We see that again the behaviour is that typical for a type II superconductor.

\subsubsection{Layered structure of the Vortices}

We can also study the properties of these vortices due to the fact that the superconducting material is composed of a stack of many not-strongly-coupled layers. We follow reference [18] where a three dimensional vortex is built up superposing a stack of two dimensional vortices in the case of a standard high $T_{c}$ superconductor. In that paper it is explicitly

computed, as a first step, the magnetic field, $\vec{b}$, produced by a single layer and then, using this result, the whole stack contribution is computed. Here we will only show that in our case we can reach, under reasonable assumptions, the same first step, and then just state the final results.

The problem has a cylindrical symmetry therefore we will use cylindrical polar coordinates $(r, \theta, z)$. The supercurrent flowing in our single layer is:

$$
\vec{J}=\left(0, J_{\theta}, 0\right) \quad J_{\theta}=J_{\theta}(r, z)=K_{\theta}(r) \delta(z)
$$

From Ampère's law we get:

$$
\vec{\nabla} \wedge \vec{b}=\vec{J} \Rightarrow\left\{\begin{array}{l}
\partial_{z} b_{r}-\partial_{r} b_{z}=K_{\theta}(r) \delta(z) \\
\partial_{z} b_{\theta}=0 \\
\frac{1}{r} \partial_{r}\left(r b_{\theta}\right)=0
\end{array}\right.
$$

from the last two we get $b_{\theta}=0$.

For $z \neq 0$ equation $\vec{\nabla} \wedge \vec{b}=0$ is solved by:

$$
A_{\theta}(r, z)=\int_{0}^{\infty} d q A_{0}(q) J_{1}(r q) e^{-q|z|} \quad A_{r}=A_{z}=0
$$


notice that $\vec{\nabla} \cdot \vec{A}=0 . A_{0}(q)$ is to be determined.

Now from the first of (4.41), solved for $z=0$, and (4.42) we can get:

$$
K_{\theta}(r)=b_{r}\left(r, 0^{+}\right)-b_{r}\left(r, 0^{-}\right)=2 \int_{0}^{\infty} d q q A_{0}(q) J_{1}(r q)
$$

For our vortex we know:

$$
K_{\theta}=\frac{e}{m}\left(\frac{n}{r}-e A_{\theta}^{e m}-A_{\theta}^{C S}\right) f^{2}(r)
$$

therefore, using (4.42):

$$
\int_{0}^{\infty} d q A_{0}(q) J_{1}(r q)\left[2 q+\frac{e^{2}}{m} f^{2}(r)\right]=\frac{e}{m} f^{2}(r)\left(\frac{n}{r}-A_{\theta}^{C S}\right)
$$

Now if the superconductor is of type II i.e. if the dimensions of the vortex are much smaller than the penetration length of $\vec{b}$, then it is sensible to take $f^{2}(r)$ equal to its mean value $v^{2}$ and $A_{\theta}^{C S}=0$. Within this approximation (4.45) becomes:

$$
\int_{0}^{\infty} d q A_{0}(q) J_{1}(r q)\left(2 q+\frac{e^{2} v^{2}}{m}\right)=\frac{e v^{2}}{m}\left(\frac{n}{r}\right)
$$

Using the orthogonality property of the Bessel functions $\int_{0}^{\infty} d r r J_{1}(r q) J_{1}\left(r q^{\prime}\right)=\frac{1}{q} \delta\left(q-q^{\prime}\right)$ we get:

$$
A_{0}(q)=\frac{e n v^{2}}{m}\left(2 q+\frac{e^{2} v^{2}}{m}\right)^{-1}=\frac{n / e}{1+\Lambda q} \quad \Lambda=\frac{2 m}{e^{2} v^{2}}
$$

$\lambda$ is related to the nominal penetration length $\lambda^{2}=\frac{m}{e^{2} \rho^{(3)}}$ by the relation:

$$
\Lambda=\frac{2 \lambda^{2}}{d}
$$

and can be considered as the two dimensional penetration length.

One can then compute (4.42) and verify that the magnetic field decays with the penetration length $\Lambda$.

These results are exactly the same obtained in [18]. We will not reproduce here all the computations that can be found in [18], but just state the main results.

Having studied what happens with a single layer we possess the building block to all the multilayered system superposing the entire stack of two dimensional vortices.

Then one can study the binding energy between vortices in different layers and find that thermal excitation breaks up the stack above a transition temperature corresponding to the Kosterlitz-Thouless temperature for a bidimensional system, see [19] [20]. 


\subsection{Magnetic field parallel to the layers}

\subsubsection{Meissner effect}

In this section we consider a different physical problem with $\vec{B}$ in the plane of the layers. This problem, for the case of standard high $T_{c}$ superconductors, has been studied in many paper by J.R. Clem and collaborators [10] [21] [22] [23]. In our analysis we choose $\vec{B}$ pointing in the $x$-direction (see Fig. $2 \mathrm{~b}$ ), and study the penetration length in $z, l_{z}$, supposing uniformity along $y$. Let us start from the following Hamiltonian:

$$
\begin{gathered}
\frac{H}{L_{y}}=\int d x d z\left\{\frac{1}{2 m d}\left|\left(\vec{\nabla}-i e \vec{A}^{e m}-i \vec{A}^{C S}\right) \phi\right|^{2}+\frac{1}{2} \vec{B}^{2}+g\left(\rho-v^{2}\right)^{2}\right\}- \\
-\frac{e^{2}}{2} \int d x d x^{\prime} d z d z^{\prime}\left\{\frac{\delta \rho\left(x^{\prime}, z^{\prime}\right)}{d} \log \left[\frac{\left(x-x^{\prime}\right)^{2}+\left(z-z^{\prime}\right)^{2}}{\Lambda^{2}}\right] \frac{\delta \rho(x, z)}{d}\right\}
\end{gathered}
$$

here we have introduced the electrostatic interaction between the fluctuations of the charged matter which, due to uniformity in the $y$-direction, is in fact the electrostatic interaction of a two-dimensional distribution of charge, which is known to be logarithmic; $\Lambda$ is a convenient dimensional constant which, since $\int \delta \rho=0$, can take an arbitrary value. In the gauge where $\vec{A}^{e m}$ and $\vec{A}^{C S}$ are both in the $y$-direction $\vec{A}^{e m}=\left(0, A^{e m}(x, z), 0\right), \vec{A}^{C S}=$ $\left(0, A^{C S}(x, z), 0\right)$ we can rewrite the Hamiltonian as:

$$
\begin{gathered}
\frac{H}{L_{y}}=\int d x d z\left\{\frac{1}{2 m d}\left(\left|\partial_{x} \phi\right|^{2}+\frac{m d^{2} j_{0}}{e}\left|\partial_{z} \phi\right|^{2}\right)+\frac{1}{2 m d}\left|e A^{e m}+A^{C S}\right|^{2} \rho+\frac{1}{2}\left(\partial_{z} A_{y}^{e m}\right)^{2}+\right. \\
\left.+g\left(\rho-v^{2}\right)^{2}\right\}-\frac{e^{2}}{2 d^{2}} \int d x d x^{\prime} d z d z^{\prime}\left\{\delta \rho\left(x^{\prime}, z^{\prime}\right) \log \left[\frac{\left(x-x^{\prime}\right)^{2}+\left(z-z^{\prime}\right)^{2}}{\Lambda^{2}}\right] \delta \rho(x, z)\right\}
\end{gathered}
$$

here $j_{0}$ is a constant depending on the intrinsic features of the material and measures the Josephson coupling between neighbouring layers.

Similarly to what we have done for the field orthogonal to the layers we consider the possible cancellation $A^{C S}(x, z)=-A^{e m}(z)\left[\theta(x) \theta\left(L_{x}-x\right)\right], L_{x}$ being the length of the $x$-edge of our sample, and $\theta$ is the step function. Thus:

$$
\delta \rho(x, z)=\frac{k}{2 \pi} \partial_{x} A^{C S}(x, z)=-\frac{k e}{2 \pi} A^{e m}(z)\left[\delta(x)-\delta\left(L_{x}-x\right)\right]
$$

The first and the last term in the first integral of (4.50) are edge effects giving a contribution which is very small compared with the rest since, going through the computation, one can 
see that they are suppressed respectively by factors $\left(L_{x}\right)^{-1}$ and $\left(L_{x}\right)^{-1 / 3}$ So in the following we will skip them. Thus:

$$
\frac{H}{L_{y}}=\frac{L_{x}}{2} \int d z\left(\partial_{z} A_{y}^{e m}\right)^{2}+\frac{k^{2} e^{4}}{4 \pi^{2} d^{2}} \int d z d z^{\prime}\left\{A^{e m}(z) \log \left[1+\frac{L_{x}^{2}}{\left(z-z^{\prime}\right)^{2}}\right] A^{e m}\left(z^{\prime}\right)\right\}
$$

Like we have done in the previous section (compare with equation (4.15)), we fix the total flux $\Phi_{0}=B L_{y} l_{z}$ and define $\varphi_{0}=\frac{\Phi_{0}}{L_{y}}$ such that for $0<z<l_{z}$ :

$$
B=\frac{\varphi_{0}}{l_{z}} \quad A^{e m}=-\frac{\varphi_{0}}{l_{z}} z
$$

and the integration is from 0 to $l_{z}$.

Therefore we get:

$$
\begin{gathered}
\frac{H}{\varphi_{0}^{2} L_{y}}=\frac{L_{x}}{2 l_{z}}+\frac{k^{2} e^{4}}{4 \pi^{2} d^{2}} \frac{1}{l_{z}^{2}} \int_{0}^{l_{z}} d z d z^{\prime}\left\{z z^{\prime} \log \left[1+\frac{L_{x}^{2}}{\left(z-z^{\prime}\right)^{2}}\right]\right\}= \\
=\frac{L_{x}}{2 l_{z}}+\frac{k^{2} e^{4}}{16 \pi^{2}} \frac{l_{z}^{2}}{d^{2}}\left[\log \frac{L_{x}^{2}}{l_{z}^{2}}+\cdots\right]
\end{gathered}
$$

We assumed $L_{x} / l_{z}$ large to expand the logarithm.

Minimizing $H$ with respect to $l_{z}$ we get:

$$
l_{z} \simeq\left(\frac{3 \pi^{2}}{k^{2} e^{4}}\right)^{1 / 3} \cdot d \cdot\left(\frac{L_{x}}{d}\right)^{1 / 3}\left[\log \left(\frac{L_{x}}{d}\right)+\cdots\right]^{-1 / 3}
$$

Therefore we have a "quasi Meissner effect" in the sense that even if $l_{z} \rightarrow \infty$ for $L_{x} \rightarrow \infty$ we have $\frac{l_{z}}{L_{x}} \rightarrow 0$. The result of equation (4.55) appears to be quite peculiar of the chiral superconductor studied in this paper.

In fact, the non-chiral superconductors would show ordinary Meissner effect for the geometry considered in this section (at least for not too small Josephson coupling between neighbouring layers): that is, magnetic fields parallel to the layers would penetrate in the direction orthogonal to the layers (say the $z$-direction) for a finite distance $l_{z}$, of the order of the standard penetration length.

The chiral superconductor studied in this paper would instead penetrate much more, for a distance $l_{z}$ as given in equation (4.55). This result holds for arbitrary Josephson coupling between neighbouring layers.

(Let us also mention that, in the case of very small Josephson coupling it is possible to imagine another configuration possibly giving a large penetration in $z$. This configuration is not peculiar of the chiral superconductor studied here. We discuss this configuration in Appendix B). 
Finally we review a general argument for multilayer superconductors indicating that the magnetic field parallel to the layers can penetrate easily in the interlayer spacing, therefore in the direction parallel to the layers (say in the $y$-direction, for a field along $x$ ), even in the case in which $l_{z}$ is finite. How much the corresponding $l_{y}$ is large, depends on how small is the interlayer Josephson coupling. This effect would be basically the same for chiral and non-chiral superconductors. In order to study this penetration in the direction parallel to the layers we follow the study of ref. [10], considering a possible vortex configuration inside the material. From this configuration we will infer the penetration properties. A crucial fact for this study is taking into account the Josephson coupling between neighbouring layers [10] [24]. This study has already been worked out in [10] for the case of a standard high $T_{c}$ superconductor, here we merely rephrase that paper for our case. The main fact is that one has to add to the supercurrent density:

$$
\vec{J}=\frac{e v^{2}}{m}(\vec{\nabla} \gamma-e \vec{A})
$$

(here $\gamma$ is the phase of $\phi$, equal to in $\theta$ for the vortex configuration), the Josephson current flowing between to neighbouring layers, say the $n$-th and the $(n+1)$-th, proportional to the sine of the gauge invariant difference of phase of $\phi$ between the layers:

$$
j_{z}=j_{0} \sin \left(\Delta \gamma_{n}\right) \quad \Delta \gamma_{n}=\gamma_{n+1}-\gamma_{n}+e \int_{n}^{n+1} d \vec{l} \cdot \vec{A}
$$

$j_{0}$ is a constant depending on the material.

Solving the Ampère equation one finds [10]:

$$
b(\tilde{r})=\frac{1}{e l_{z} \lambda_{J}} K_{0}(\tilde{r}) \quad \tilde{r}=\sqrt{\frac{y^{2}}{\lambda_{J}^{2}}+\frac{z^{2}}{l_{z}^{2}}}
$$

with $\lambda_{J}^{2}=\frac{1}{e d j_{0}}$; here $d$ is the stack periodicity of the layers.

This result tells essentially that $\vec{B}$ penetrates differently along $y$, i.e. parallel to the layers, and along $z$, i.e. orthogonally, in other words the vortex has an "elliptic symmetry". Notice that in reference [10] the decay length along the $y$-axis is called $\lambda_{z}$ instead of $\lambda_{J}$; the reason is that this decay length is essentially due to the screening currents which are pointing in the orthogonal direction i.e. the $z$-direction. Similar argument goes for the decay length along the $z$-axis which in [10] is called $\lambda_{y}$.

In conclusion, in both cases of ordinary and chiral superconductors the magnetic field would easily penetrate along the interlayer spacing. Instead, the penetration across the layers of a magnetic field parallel to them will be finite for an ordinary superconductor (for not too small Josephson coupling), whereas it will grow with a fractional power of the sample's dimension for the chiral superconductor. 


\section{Appendix A.}

In the standard Chern-Simons (CS) description of Anyons one introduces a CS gauge field $\vec{A}$, whose field strength is proportional to the density:

$$
\vec{\nabla} \wedge \vec{A} \propto \rho
$$

In this formulation to have translational invariance one has to take care of the boundary conditions in a proper way and to take a torus [6] [7] [8] [9]. In this way one shows that the ground state of the full quantum solution of the mean field theory corresponds to a constant density. Due to the non-trivial topological properties of the torus one has to take into account the topological components of the gauge potential (also called "flat connections" since $\vec{\nabla} \wedge \vec{a}=0$ ) defined by:

$$
a_{x}=\oint d x A_{x} \quad a_{y}=\oint d y A_{y}
$$

here the two integrals are performed along the two non-trivial loops of the torus. In this way the Hamiltonian can be written as follows [6] [7] [8] [9]:

$$
H=\int d^{2} x \int d^{2} a\left\{\frac{1}{2 m}|\vec{D} \psi|^{2}+c\left|\left(\frac{k}{4 \pi} a_{i}+i \epsilon_{i j} \frac{\partial}{\partial a_{j}}\right) \psi\right|^{2}\right\}
$$

where the covariant derivative is:

$$
D_{i}=\partial_{i}-\frac{i \pi}{2 k} v^{2} \epsilon_{i j} x_{j}-i \frac{a_{i}}{L}-i \tilde{A}_{i}
$$

here $L$ is the length of a side of the torus (we are supposing, for simplicity that our torus is a square with identified edges), $v^{2}$ is the mean density, $\overrightarrow{\tilde{A}}$ is the fluctuation part of $\vec{A}$ such that $\vec{\nabla} \wedge \overrightarrow{\tilde{A}} \propto \delta \rho . c$ is a positive constant whose value can be arbitrary in what follows. In the Anyon problem the value of $c$ is large, $c \rightarrow \infty$, and thus the $a_{i}$ degrees of freedom remain in the ground state.

In the case of the infinite plane we have to take the limit $L \rightarrow \infty$ at constant density (we will call it thermodynamical limit), so $\frac{a_{i}}{L} \rightarrow 0$ if $a_{i}$ is bounded (we will see below that indeed $a_{i}$ is bounded) but in the Hamiltonian still survives a term in $a_{i}$. 
Note that now the wavefunction $\psi$ is now a function of $\vec{a}$ beside $\vec{x}$; i.e. $\psi=\psi(\vec{x}, \vec{a})$ so we define the density $\rho(\vec{x})$ to be:

$$
\rho(\vec{x})=\int d^{2} a|\psi(\vec{x}, \vec{a})|^{2}
$$

It is convenient to introduce a complex notation:

$$
A=A_{M}-i \tilde{A} \quad \bar{A}=\bar{A}_{M}+i \overline{\tilde{A}}
$$

where

$$
A_{M}=\frac{i}{2} \frac{\pi}{k} v^{2} \bar{z} \quad \bar{A}_{M}=-\frac{i}{2} \frac{\pi}{k} v^{2} z
$$

are the "mean field" parts and

$$
\tilde{A}=\frac{i}{2}\left(\tilde{A}_{1}-i \tilde{A}_{2}\right) \quad \overline{\tilde{A}}=-\frac{i}{2}\left(\tilde{A}_{1}+i \tilde{A}_{2}\right)
$$

are the "fluctuating" parts; for the flat connections we define:

$$
a=\frac{1}{2 \pi}\left(i a_{1}+a_{2}\right) \quad \bar{a}=\frac{1}{2 \pi}\left(-i a_{1}+a_{2}\right)
$$

For the $C S$ magnetic field we have:

$$
B=2 i(\bar{\partial} A-\partial \bar{A})=B_{M}+\tilde{B}=-\frac{2 \pi}{k} \rho
$$

where:

$$
B_{M}=-\frac{2 \pi}{k} v^{2} \quad \tilde{B}=(\bar{\partial} \tilde{A}-\partial \overline{\tilde{A}})=\frac{2 \pi}{k}\left(v^{2}-\rho\right)=-\frac{2 \pi}{k} \delta \rho .
$$

Let us now suppose that $\tilde{A}=0$ and study the mean field solution. The Hamiltonian is:

$$
H=\int d^{2} z \int d^{2} a\left\{\frac{2}{m}\left|\left(\partial+\frac{\pi}{2 k} v^{2} \bar{z}\right) \psi\right|^{2}+\frac{\pi}{m k} v^{2}|\psi|^{2}+\frac{c}{\pi^{2}}\left|\left(\frac{\partial}{\partial a}+\frac{\pi k}{2} \bar{a}\right) \psi\right|^{2}\right\}
$$

The state of minimal energy corresponds to:

$$
\psi=\psi_{M}=e^{-\frac{\pi v^{2}}{2 k} z \bar{z}-\frac{\pi k}{2} a \bar{a}} g(\bar{z}, \bar{a})
$$

here $g$ is an arbitrary antiholomorphic function.

From (A.13) we can see that $a$ is bounded.

Let us look for the constant density solutions, which are known to correspond to the ground state of the full quantum mechanical mean field problem. Choosing $g(\bar{z}, \bar{a})=e^{\pi v \bar{z} \bar{a}} v$ we get $\rho_{M}=v^{2}$, in fact

$$
\rho_{M}=\int d^{2} a\left|\psi_{M}\right|^{2}=\int d^{2} a e^{-\pi k\left|a-\frac{v}{k} \bar{z}\right|^{2}} v^{2}=v^{2}
$$


provided we normalize the measure $d^{2} a$ such that $\int d^{2} a e^{-\pi k a \bar{a}}=1$.

For the Hamiltonian (A.12) we get:

$$
H_{M}=\frac{\pi}{m k} v^{2} N
$$

this is the energy of $N$ particles in the lowest Landau level. We know that in the mean field solution actually the $N$ particles fill exactly $k$ levels corresponding to the energy:

$$
E_{M}=\frac{1}{2 m}\left|B_{M}\right| N k
$$

So, in order to reproduce the correct mean field energy, we must add:

$$
E^{\prime}=\frac{1}{2 m}\left|B_{M}\right| N(k-1)=\frac{2 \pi}{m}\left(1-\frac{1}{k}\right) \int d^{2} a \int d^{2} z|\psi|^{4}
$$

So our correct starting Hamiltonian is:

$$
H=\int d^{2} x \int d^{2} a\left\{\frac{1}{2 m}|\vec{D} \phi|^{2}+\frac{c}{\pi^{2}}\left|\left(\frac{\partial}{\partial a}+\frac{\pi k}{2} \bar{a}\right) \psi\right|^{2}+\frac{2 \pi}{m}\left(1-\frac{1}{k}\right)|\psi|^{4}\right\}
$$

Notice that more in general a constant density is also obtained taking:

$$
\psi_{M}=e^{-\frac{\pi v^{2}}{2 k} z \bar{z}-\frac{\pi k}{2} a \bar{a}+\pi v \bar{z} \bar{a}+i(p z+\bar{p} \bar{z})} v
$$

then the Hamiltonian (A.18) becomes:

$$
H_{M}=\int d^{2} z\left\{\frac{2}{m}|i p|^{2} v^{2}-\frac{k}{2 m} B_{M} v^{2}\right\}=\frac{1}{2 m}\left(p_{x}^{2}+p_{y}^{2}\right) N+\frac{k}{2 m}\left|B_{M}\right| N
$$

so we have found, beside the standard mean field energy (A.16) a kinetic energy equal to that of one particle times $N$. So our system moves like a condensate where all particles have the same momentum. In other words it represents a collective motion. If we compute the currents:

$$
J=\int d^{2} a \frac{1}{2 m i}\left[\psi^{\dagger} D \psi-\psi(D \psi)^{\dagger}\right]
$$

we get:

$$
J=\frac{v^{2}}{m} p
$$

So we have found for the current exactly the charge density times the velocity.

Now let us introduce the fluctuations taking $\tilde{A} \neq 0$ :

$$
\begin{aligned}
H=\int d^{2} z \int d^{2} a\left\{\frac{2}{m} \mid(\partial\right. & \left.+\frac{\pi}{2 k} v^{2} \bar{z}-\tilde{A}\right)\left.\psi\right|^{2}-\frac{1}{2 m} B|\psi|^{2}+\frac{2 \pi}{m}\left(1-\frac{1}{k}\right)|\psi|^{4}+ \\
& \left.+\frac{c}{\pi^{2}}\left|\left(\frac{\partial}{\partial a}+\frac{\pi k}{2} \bar{a}\right) \psi\right|^{2}\right\}
\end{aligned}
$$


Then if we take:

$$
\psi=e^{-\frac{\pi v^{2}}{2 k} z \bar{z}-\frac{\pi k}{2} a \bar{a}+\pi v \bar{z} \bar{a}} \phi(z, \bar{z})
$$

for the density we get:

$$
\rho=\int d^{2} a|\psi|^{2}=\int d^{2} a e^{-\pi k\left|\bar{a}-\frac{v}{k} z\right|^{2}}|\phi(z, \bar{z})|^{2}=|\phi(z, \bar{z})|^{2}
$$

and for the Hamiltonian:

$$
H=\int d^{2} r\left\{\frac{1}{2 m}|(\vec{\nabla}-i \overrightarrow{\tilde{A}}) \phi|^{2}+g|\phi|^{4}\right\}
$$

where in order to reproduce the correct "mean field" energy we have to take $g=\frac{\pi}{m}\left(1-\frac{1}{k}\right)$.

So we have found an Hamiltonian which in the "mean field" case gives the correct answer and in the more general "fluctuating" case recovers the covariant derivatives taking into account the density fluctuations.

We take equation (A.26) as the basic effective Hamiltonian, describing our quantum fluid, corresponding to the effective Lagrangian density (2.1). 


\section{Appendix B.}

We consider the geometry of section 4.2, Fig. $2 b$ ), and a configuration relevant for the penetration of the magnetic field in the $z$-direction.

Assume that the system is homogeneous in the $x$-direction. We suppose that $\phi$ is constant up to a phase i.e. $\phi=v e^{i \gamma}$, therefore we have no electrostatic interaction and put $A^{C S}=0$ (therefore this configuration would be there also in a non chiral superconductor).

In this configuration one considers the possibility that the phase is responsible for the cancellation of $A^{\text {em }}$ :

$$
\partial_{y} \gamma=e A^{e m}
$$

We again suppose to have a fixed external flux so that equation (4.53) still holds.

We further impose periodic boundary conditions (ensuring the current conservation):

$$
\gamma(0)=\gamma\left(L_{y}\right)=0(\bmod 2 \pi)
$$

From (B.1) and (B.2) we get:

$$
e L_{y} A^{e m}=2 \pi p
$$

here $p \in \mathbf{Z}$ has in general a different value in different layers. Since $d$ is the spacing between the layers the $z$ coordinate of the $s$-th layer is $z_{s}=s d$. Now recalling equation (4.53) we get:

$$
e L_{y} A^{e m}\left(z_{s}\right)=-e L_{y} \frac{\varphi_{0}}{l_{z}} s d=2 \pi p_{s} \quad \Rightarrow \quad p_{s}=-\frac{e}{2 \pi} \frac{d}{l_{z}} \varphi_{0} L_{y} s \in \mathbf{Z}
$$

but since the total flux is quantized $\varphi_{0} L_{y}=\frac{2 \pi}{e} n$ we have:

$$
\frac{p_{s}}{s}=-\frac{d}{l_{z}} n
$$

since $\frac{l_{z}}{d}$ is the number of layers "penetrated" by $B$ this means that every layer bears an identical fraction of the total flux.

The Hamiltonian of this system can be written as:

$$
H=\int d x d y d z\left\{\frac{1}{2 e^{2} \lambda^{2}}\left|\partial_{y} \gamma-e A^{e m}\right|^{2}+\frac{1}{2 e^{2} \lambda_{J}^{2}}\left|\partial_{z} \gamma\right|^{2}+\frac{1}{2}\left(\partial_{z} A^{e m}\right)^{2}\right\}
$$


the second term is the Josephson coupling between neighbouring layers [24]. $\lambda_{J}$ is a constant of the dimension of a length whose physical significance will be clear in the following, it is related to the Josephson maximal current density $j_{0}$ by the equation:

$$
j_{0}=\frac{1}{e d \lambda_{J}^{2}}
$$

$\lambda$ is the standard penetration length (4.14). Since from (B.1) we have $\gamma=e y A^{e m}$, we can rewrite the hamiltonian as:

$$
H=L_{x} \int_{0}^{L_{y}} \int_{0}^{l_{z}} d z\left\{\frac{1}{2}\left(\partial_{z} A^{e m}\right)^{2}\left(1+\frac{y^{2}}{\lambda_{J}^{2}}\right)\right\}=\frac{1}{2} \varphi_{0}^{2} \frac{L_{x} L_{y}}{l_{z}}\left[1+\frac{1}{3}\left(\frac{L_{y}}{\lambda_{J}}\right)^{2}\right]
$$

which is minimal for $l_{z}$ macroscopic i.e. for $l_{z} \sim L_{z} \sim L_{y} \sim L_{x}$. To see if this configuration is favoured one has to compare it with the energy of the standard case in which:

$$
H^{\text {standard }}=\varphi_{0}^{2} \frac{L_{x} L_{y}}{\sqrt{3} \lambda}
$$

That is, for $l_{z} \sim L_{y}$, the configuration giving (B.8) is favourite if:

$$
\frac{1}{\sqrt{3} \lambda}>\frac{1}{2 l_{z}}\left[1+\frac{1}{3}\left(\frac{L_{y}}{\lambda_{J}}\right)^{2}\right]
$$

For all common high $T_{c}$ materials $\lambda_{J} \simeq 10^{5} \AA$ (corresponding to $j_{0} \simeq 10^{4} \mathrm{~A} / \mathrm{cm}^{2}$, see for example [25]) therefore since $\frac{\lambda_{J}}{\lambda} \simeq 120$, for $l_{z} \sim L_{y} \lesssim 10^{8} \AA$, this configuration could be marginally competitive. When equation (B.10) is not satisfied then this configuration would not be favourite. 


\section{Figure Captions}

1. Qualitative spectrum.

2. Directions of the magnetic field with respect to the layers. 


\section{References}

1. S.C. Zhang, T.H. Hansson, S. Kivelson, Phys. Rev. Lett. 62 (1989), 82.

2. D.H. Lee, S.C. Zhang, Phys. Rev. Lett. 66 (1991), 1220.

3. D.H. Lee, M.P.A. Fisher, Int. J. Mod. Phys. B5 (1991), 2675.

4. S.C. Zhang, Int. J. Mod. Phys. B6 (1992), 25.

5. D. Boyanovsky, Int. J. Mod. Phys. A7 (1992), 5917.

6. R. Iengo, K. Lechner, Nucl. Phys. B346 (1990), 551.

7. R. Iengo, K. Lechner, Dingping Li, Phys. Lett. B269 (1991), 109.

8. R. Iengo, K. Lechner, Phys. Rep. 213 (1992), 179.

9. R. Iengo, K. Lechner, Nucl. Phys. B384 (1992), 541.

10. J.R. Clem, M.W. Coffey, Phys. Rev. B42 (1990), 6209.

11. S.K. Paul, A. Khare, Phys. Lett. B174 (1986), 420.

12. J. Hong, Y. Kim, P.Y. PAC, Phys. Rev. Lett. 64 (1990), 2230.

13. R. Jackiw, E.J. Weinberg, Phys. Rev. Lett. 64 (1990), 2234.

14. E.B. Bogomol'Nyi, Sov. J. Nucl. Phys. 24 (1976), 449.

15. R. Jackiw, S.Y. Pi, Phys. Rev. Lett. 64 (1990), 2969.

16. X.G. Wen, A.Zee, Phys. Rev. Lett. 62 (1989), 2873.

17. L.N. Bulaevskit, Int. J. Mod. Phys. B4 (1990), 1849.

18. J.R. Clem, Phys. Rev. B43 (1991), 7837.

19. J.M. Kosterlitz, D. Thouless, J. Phys. C6 (1973), 1181.

20. M.R. Beasley, J.E. Mooij, T.P. Orlando, Phys. Rev. Lett. 42 (1979), 1165.

21. J.R. Clem, M.W. Coffey, Z. HaO, Phys. Rev. B44 (1991), 2732.

22. L. Bulaevskit, J.R. Clem, Phys. Rev. B44 (1991), 10234.

23. J.R. Clem, Physica C 162-164 (1989), 1197. 
24. W.E. Lawrence, S. Doniach, in "Proceedings of the XII International Conference on Low Temperature Physics" (E. Kanda, Ed.), p.361, Academic Press, Tokio, 1971.

25. J.R. CLEM, in "Physics and materials science of high temperature superconductors" (R. Kossowski, S. Methfessel, D. Wohlleben, Eds.) NATO ASI Series E Vol. 181, p. 79, Kluwer Academic Publishers, Dordrecht, 1989. 